\title{
Electric charge limits on settled powders
}

Cite as: J. Appl. Phys. 119, 223302 (2016); https://doi.org/10.1063/1.4953649

Submitted: 17 March 2016 . Accepted: 28 May 2016 . Published Online: 13 June 2016

J. Pérez-Vaquero, M. A. S. Quintanilla (iD, and A. Castellanos

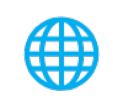

View Online

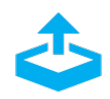

Export Citation

\section{ARTICLES YOU MAY BE INTERESTED IN}

Triboelectric charging of insulating polymers-some new perspectives

AIP Advances 2, 010701 (2012); https://doi.org/10.1063/1.3687233

New instrument for tribocharge measurement due to single particle impacts

Review of Scientific Instruments 78, 024706 (2007); https://doi.org/10.1063/1.2671585

Effect of humidity on contact electrification due to collision between spherical particles

AIP Advances 6, 035117 (2016); https://doi.org/10.1063/1.4944831

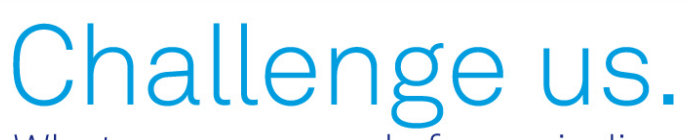

What are your needs for periodic signal detection?

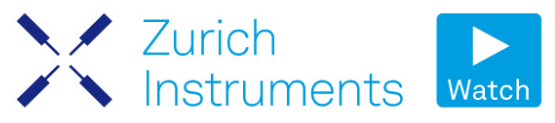

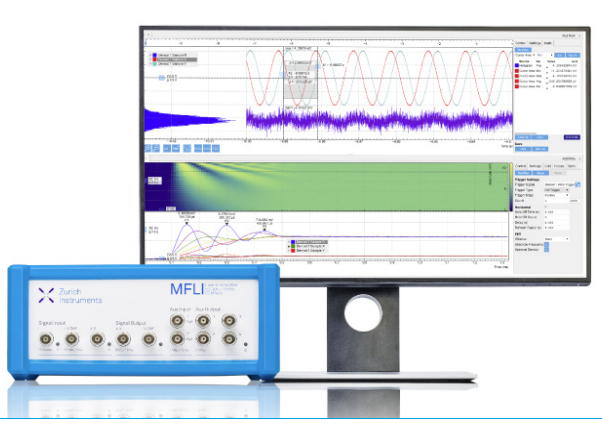

119, 223302 


\title{
Electric charge limits on settled powders
}

\author{
J. Pérez-Vaquero, M. A. S. Quintanilla, ${ }^{\text {a) }}$ and A. Castellanos \\ Department of Electronics and Electromagnetism, Faculty of Physics, Universidad de Sevilla, Sevilla, Spain
}

(Received 17 March 2016; accepted 28 May 2016; published online 13 June 2016)

\begin{abstract}
In flows of dry particulate systems, electric charge is generated on particle surfaces by their collision with walls and with other particles. Charge build-up on single particles can yield local charge values high enough to surpass the limiting electric field for corona discharge into the surrounding gas. Then, local charge is decreased to a lower value that becomes stabilized when flows stop and particles deposit in a container. In this paper, we have used a Faraday pail system to measure the residual particle charge after using two different devices-tribochargers-for particle charging. One of the tribochargers allowed us to directly measure the total charge that was transferred from the walls to the particles, and this was compared to the final values in the bulk powder once it was collected in the Faraday pail. The results show that the electric charge of particles dispersed in gas is limited by corona discharge and depends mainly on the particle size. In addition, we present a simple model of the discharge of the collected powder based on electrostatic considerations. If the powder effective conductivity and the electric charge of the settling particles are known, the model predicts the temporal evolution of the total charge of the collected powder and the spatial distribution of the electric charge and electric field. Published by AIP Publishing.
\end{abstract}

[http://dx.doi.org/10.1063/1.4953649]

\section{INTRODUCTION}

Dry powder handling operations are usually accompanied by electric charge built-up due to triboelectrification, sometimes also called contact charging. Particle contact charging can be beneficial in some cases, such as electrostatic precipitation and xerographic printing, or detrimental, for example, in pneumatic conveying. The physical processes that determine the magnitude of the charge transferred between two surfaces have attracted interest for a long time, and while the physics of triboelectrification between the conductive surfaces is well understood, there is no well established theory that explains triboelectrification between nonconductive surfaces. While for conductive materials the charge transferred between surfaces in contact results from electron transfer driven by the differences in Fermi levels; ${ }^{1,2}$ when at least one of the surfaces in contact is not conductive, the resulting charge transfer has been explained using an effective work function for the non-conductive solid, ${ }^{3,4}$ as a consequence of the exchange of mobile ions ${ }^{5}$ or the existence of donors and acceptor centers representing localized electron sites on the surfaces of the solids in contact. ${ }^{6,7}$ In any case, a distinction has to be made between the charge transferred between two surfaces when they are in contact and the charge remaining in the surfaces after separation. When two surfaces charged with different polarities are separated, the electric field between both surfaces increases with increasing separation, up to the point when some charge can be back transferred to its original surface. ${ }^{8}$ The practical consequence of this fact for powder handling is that the maximum charge than an isolated particle can hold must be

\footnotetext{
a) Author to whom correspondence should be addressed. Electronic mail: quintani@us.es
}

limited by the electrical breakdown field in its surrounding gas, rather than by the physical mechanisms that caused the charge transfer between the particle and the solid surface from where the charge was picked by the particle while in contact. Indeed, it is known that the charge transferred between non-conductive polymers in vacuum is much larger than in the atmosphere. ${ }^{9}$ However, in most powder handling processes, particles cannot be considered as isolated and the total charge present in a powder is also limited by spatial charge effects. In pneumatic transport, spatial charge effects are not relevant unless the volume fraction of dispersed particles is greater than a certain limit that depends on particle size and pipe diameter, ${ }^{10}$ but when the particles settle to form a packed powder, the concentration of charge can create electric fields large enough to trigger electrical discharges. The type of discharge that occurs depends on the depth of the packed powder. For depths of the order of a meter, the total charge accumulated can trigger a type of electrical discharges known as cone discharges ${ }^{11}$ in which the electrical breakdown of the surrounding air occurs through the formation of a conductive channel that carries the charge from the powder to its surroundings: typically, the walls of the silo holding the powder. ${ }^{12}$ However, in the initial stages of the formation of heap of powder, there is no enough charge accumulated to form a conductive channel and it is expected that a corona discharge from the surface of the powder to the surrounding air takes place, as it happens in some situations in electrostatic powder coating. ${ }^{13}$ The charge remaining in the powder would then be limited to the amount necessary to create the electric field to maintain the corona discharge. In this work, we have measured both the charge acquired by the powder during pneumatic transport and the electrical charge remaining in the powder once collected. 


\section{EXPERIMENTAL SETUP}

In our experiments, powders are first dispersed in a gas stream and acquire electrical charge through collisions with a tribocharger. Once they come from a tribocharger, the powder is collected in a Faraday pail where the mass and charge of the collected powder are measured as a function of time. A photo of the setup is shown in Fig. 1. The dispersion procedure depends on whether the powder is fluidizable or not (the list of powder tested and their properties are given at the end of this section). For free-flowing materials, a small silo with an adjustable hole on its bottom is used for gravity unloading of the powder into the suction port of a venturi. For non free-flowing materials, the silo is replaced by a closed cell with a porous plate subjected to mechanical vibration and blown with a constant gas flow. The particles which are elutriated from the sample go into the suction port of the venturi. In both cases, the mass flow rate is kept as constant as possible. The typical value of gas flow rates exiting the venturi yields input gas velocities in

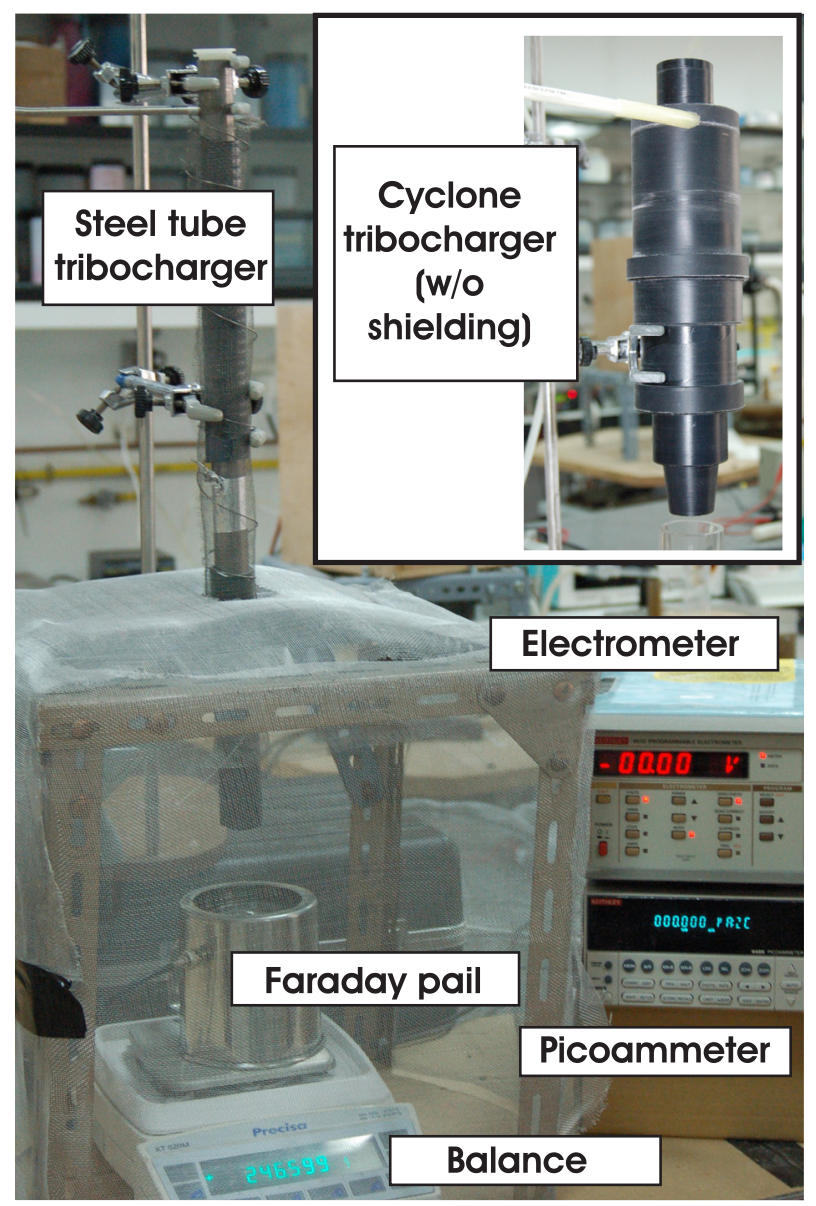

FIG. 1. The experimental setup for measuring triboelectrification levels, with the steel tube tribocharger in place and the nylon cyclone tribocharger in the inset, shown without electric shielding. The Faraday pail resting on the balance collects the powder coming from the tribocharger. The electric current flowing into the Faraday pail is measured by the picoammeter and recorded by the PC. When the steel pipe is used as tribocharger, the electrometer depicted in the figure is connected to it and its data are also registered by the PC. The metallic mesh around all the setup is grounded to reduce electric noise from the rest of the lab. The dispersion units do not appear in the figure. the tribochargers that range from 10 to $20 \mathrm{~m} / \mathrm{s}$ for all the measurements we made.

We have made experimental runs with two different tribochargers: a nylon cyclone $(350 \mathrm{~mm}$ in length, $74 \mathrm{~mm}$ internal diameter) and a steel tube (length $540 \mathrm{~mm}$ and internal diameter $21 \mathrm{~mm}$ ). In both cases, the inlet port of the tribocharger is tangent to its walls to improve the collision rate. Cyclone tribochargers have been used by other authors ${ }^{14}$ due to their ability to separate the particles from the gas stream, although particle-gas separation is increasingly inefficient for particles with sizes close to or below $10 \mu \mathrm{m}$. The steel tube tribocharger does not separate the particles from the gas but, being made of a conductive material, it allows us to measure the electric charge $Q_{d}$ transferred to the particles while they are dispersed into the gas stream. To this aim, a programmable electrometer (Keithley 6512) working in the ammeter mode connects ground to the tribocharger and measures the electric current to the steel pipe. $Q_{d}$ is obtained by numerical integration of the registered current. In order to reduce the noise pickedup by capacity coupling, an electrically grounded metallic mesh covers the pipe. The mesh and the pipe are insulated form each other and grounded by separate connections. The use of one tribocharger made of conductive material and another tribocharger of non-conductive material allows us to probe if the microscopic mechanism of the charge transfer between the particles and the walls of the tribocharger has any effect on the charge acquired by particles dispersed in the gas stream.

The powder that exits the tribocharger settles by gravity into a cylindrical cell whose walls are made of insulating material (a methacrylate tube), and it is closed at its bottom with a metallic filter to help separate the particles from the gas. The filter inside cell is electrically insulated to the outside of the cell. The cell is located inside the Faraday pail consisting of an inner and an outer cage made of conductive material, the former being connected to ground through a picoammeter (Keithley 6485). The picoammeter measures the current flowing from ground to the inner cage of the Faraday pail as the collecting cell is filled by the material exiting the tribocharger. Integration of this current yields the charge $Q_{s}(t)$ in the settled powder as a function of time. The outer cage is grounded to act as a shield to external interferences, and in some runs, it was supplemented by a grounded metallic grid around all the Faraday pail acting as a second shield. Note that we make a distinction between the electric charge $Q_{d}$ acquired by the powder when dispersed and the electrical charge $Q_{s}$ remaining in the particles when settled, because the charges $Q_{d}$ and $Q_{s}$ are not necessarily the same. The Faraday pail system rests on a mass balance (Mettler Toledo AB204-S or Precisa XT series, depending on the mass involved) that measures the collected powder mass $m(t)$ as a function of time.

Both mass and electric intensity data are registered in a PC (personal computer) at a rate of 4-10 samples per second. Uncertainties in the values of the charge are calculated from the root mean square amplitude of the noise voltage in the ana$\log$ output channels of the picoammeter and the electrometer. 


\section{MATERIALS}

Different materials have been used to cover a range of particle sizes as large as possible: poly-(methyl methacrylate) (PMMA) beads (Polysciences, Inc.), 5-50 $\mu \mathrm{m}$ glass microspheres (Duke Scientific Corp.), 70-100 $\mu \mathrm{m}$ and 90-150 $\mu \mathrm{m}$ glass beads (Sigmund-Lindner $\mathrm{GmbH}$ ), commercial sugar, commercial semoline, and cornstarch (Maizena ${ }^{\circledR}$ ). Particle size analysis of every material but PMMA beads (whose average size was taken from the manufacturer data sheet) was performed with a laser diffraction analyzer (Mastersizer Scirocco 2000), by dry dispersion in air (1 bar pressure) according to ISO 13320. The mean surface (Sauter mean) diameter of these materials is listed in Table I. The conditions and the results for the tests on each material are listed in Table I. In some cases, the sample tested was stored at controlled ambient conditions at two different relative humidities $(30 \%$ and $60 \%$ $\mathrm{RH})$ before each test to investigate the effect of storage humidity on the electrical charge picked by the powder.

\section{RESULTS}

\section{A. Charge per particle in suspension}

The charge acquired by the particles in the tribocharger while they are dispersed in the gas stream $q_{d}$ can only be evaluated for the steel tube tribocharger, for which the total charge $Q_{d}$ given to the powder can be measured. An example of such measurement is shown in Fig. 2, where $Q_{d}$ is presented as a function of the mass $m$ collected in the Faraday pail. In all experiments performed, the plot of $Q_{d}$ against $m$ has a linear dependence with the mass $m$, as shown in Fig. 2. This fact indicates that, assuming the sample is monodisperse, the particles acquire a constant charge $q_{d}=Q_{d} m_{p} / m$ from the steel tube for the duration of the experiment, where $m_{p}$ is the particle mass. Fig. 3 shows the value of $q_{d}$ as a function of the particle radius $r_{p}$ (half the surface mean diameter listed in Table I) for the experiments using the steel tribocharger. Since there is always some powder that remains stuck to the inner walls of the tribocharger, using the collected mass $m$ somewhat overestimates the value of $q_{d}$. The typical mass loss ranges from $19 \%$ to $69 \%$ of the total mass of dispersed powder for 5-50 $\mu \mathrm{m}$ glass beads and $1 \%-20 \%$ in $90-150 \mu \mathrm{m}$. In general, the mass loss decreases with larger particles and higher storage humidity.

Such overestimation in charged mass has impact on two process variables, $q_{d}$ and volume fraction of powder, $\psi$, as

TABLE I. Materials used in the experiments presented in this paper. Their mean particle size (surface-mean diameter) was measured in a Mastesizer 2000 using a dry dispersion module (air, dispersion pressure 1 bar).

\begin{tabular}{lc}
\hline \hline Material & Surface mean diameter $(\mu \mathrm{m})$ \\
\hline Cornstarch & 7.3 \\
$5-50$ glass beads & 33.9 \\
$70-110$ glass beads & 92.0 \\
$90-150$ glass beads & 125.4 \\
PMMA beads & 200 \\
Sugar & 720 \\
Semoline & 796 \\
\hline \hline
\end{tabular}

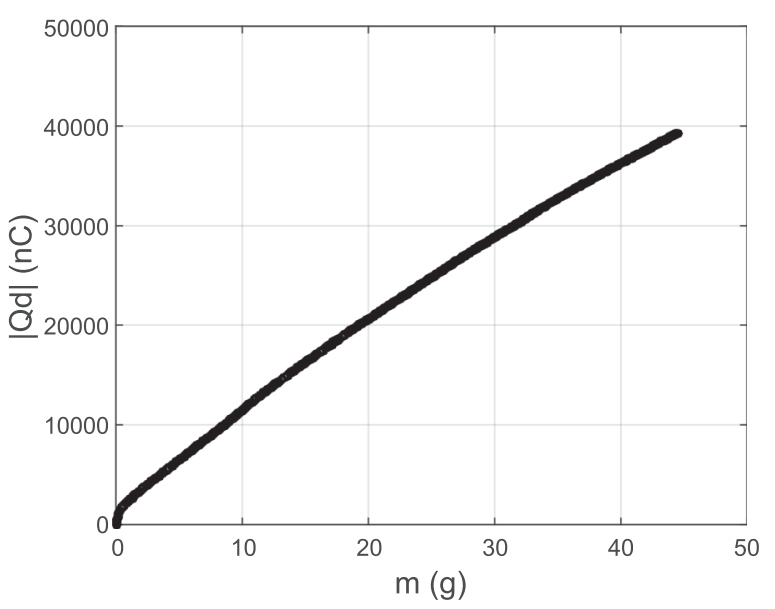

FIG. 2. Electric charge $Q_{d}$ ceded by the steel tribocharger to PMMA beads (storage humidity not controlled) as a function of the collected mass $m$ in the Faraday pail. The linear dependence of $Q_{d}$ with $m$ indicates that the particles are charged uniformly when the pass through the tribocharged.

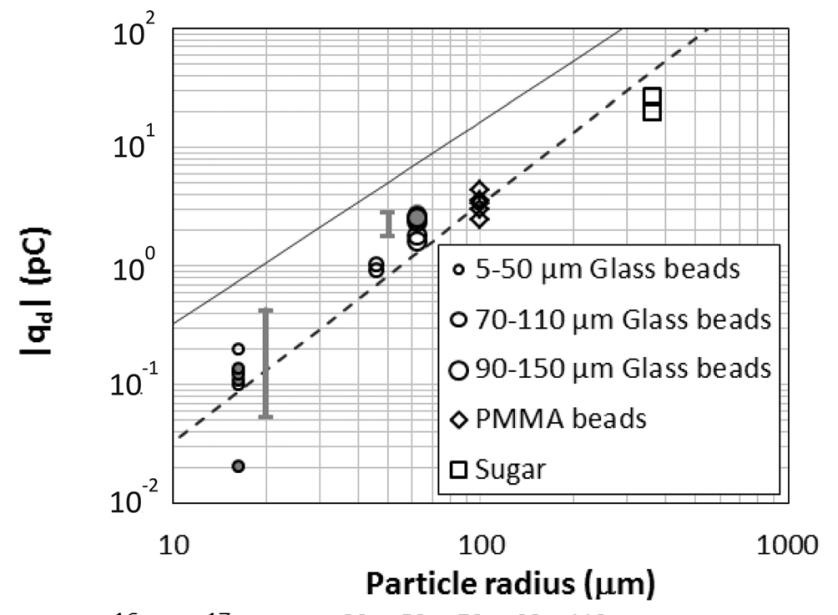

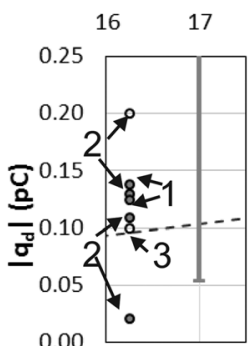

- 5-50 $\mu \mathrm{m}$ Glass beads

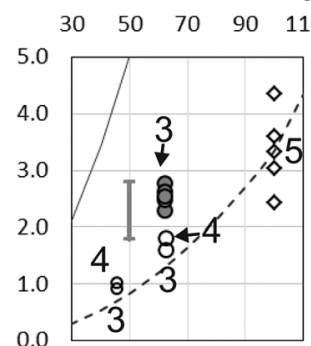

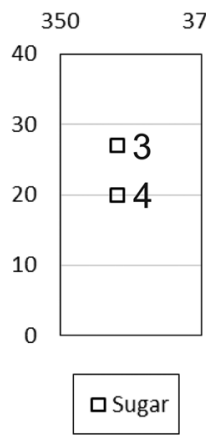

FIG. 3. Results of absolute values of transferred charge $q_{d}$ from the steel pipe tribocharger to particles of different materials. Plotted lines are the theoretical values for maximum charge of particles limited by planar corona discharge (dotted line) and size dependent corona discharge (solid line), Eq. (2). The dispersing gas and storage conditions of the material at each experimental point are given according to the following code: 1: Dispersed in $\mathrm{N}_{2}$, stored at $10 \% \mathrm{RH} ; 2$ : Dispersed in $\mathrm{N}_{2}$, stored at $60 \% \mathrm{RH} ; 3$ : Dispersed in air, stored at $60 \% \mathrm{RH}$; 4: Dispersed in air, stored at 30\% RH; and 5: Dispersed in air, no humidity control during storage. For experiments in which the collected mass $m_{d}$ was available (filled symbols), the particle charge $q_{d}$ was calculated using the dispersed mass $m_{d}$ rather than the collected mass $m$. Error bars indicate the typical uncertainty for each size range. For sugar particles, error bar and symbol are approximately the same size. Subplots in linear scale zoom into areas of interest of the main plot. 
shown in Figures 3, 4, and 8. In these figures, grey coloured bars by each group of symbols indicate the uncertainty in either $q_{d}, \psi$, or both, when mass losses between $19 \%$ and $69 \%$ are considered for the 5-50 $\mu \mathrm{m}$ glass beads, and $20 \%$ for the 90-150 $\mu \mathrm{m}$ glass beads. The bar corresponding to a mass loss of $1 \%$ is neglected since its effect is not visually noticeable.

In Fig. 3, we have drawn lines for the maximum particle charge $q_{d, \text { max }}$, assuming the value of $q_{d, \text { max }}$ is given by the condition that the electric field on the particle surface equals the breakdown field for corona discharge as suggested in Ref. 8. We have plotted two lines: one (dashed line in Fig. 3) assuming this field is equal to $E_{c}=3 \times 10^{6} \mathrm{~V} / \mathrm{m}$ irrespective of particle size, and another line (solid line in Fig. 3) assuming its value is particle size dependent, as given in Ref. 1

$$
E_{c}=9.29 \times 10^{5} r_{p}^{-0.3}
$$

where $\epsilon_{o}$ is the electric permittivity of the gas surrounding the particle, which we assume is equal to the permittivity of vacuum $\left(\epsilon_{o}=8.85 \times 10^{-12} \mathrm{~F} / \mathrm{m}\right)$. According to this, the maximum charge for an isolated particle in air, assuming a size-dependent corona discharge, is given by

$$
q_{d, \max }=1.03 \times 10^{-4} r_{p}^{1.7}
$$

where $q_{d, \text { max }}$ is measured in coulombs and $r_{p}$ in meters. All the experimental data lie very close to the corona discharge line which considers a constant critical electric field. Thus, the data support the fact that, when dispersed, the particles charge up to their maximum attainable value.

For each material presented in Fig. 3, several RH values at storage have been used and, in the case of the finest glass beads, different dispersion gases (air and dry $\mathrm{N}_{2}$ ). The conditions on humidity and dispersing gas for each

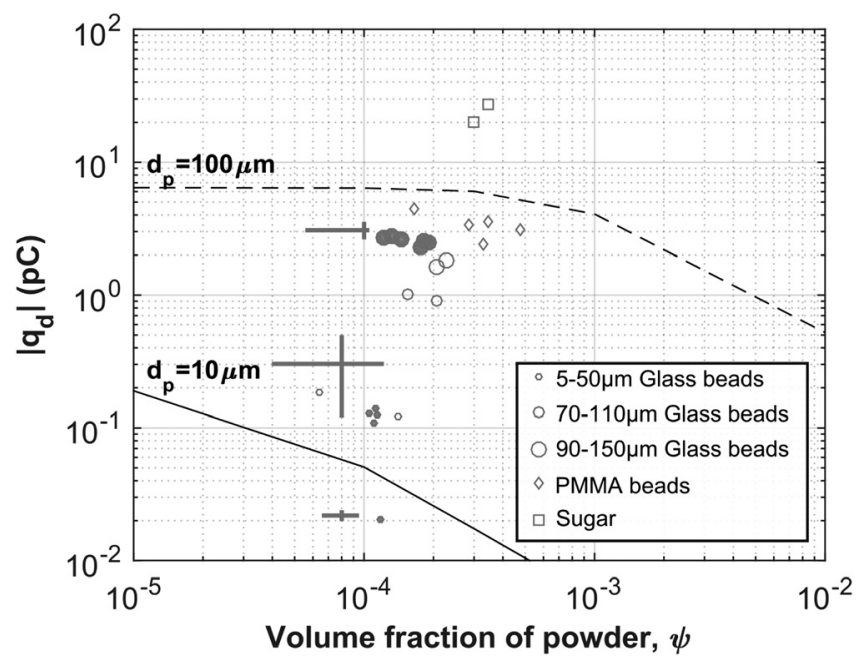

FIG. 4. Transferred charge per particle $q_{d}$ in the steel tribocharger as a function of the volume fraction of the powder in the dispersing gas stream $\varphi$. As a reference, the lines represent the maximum charge per particle when the effect of the charge of each particle on its neighbors is taken into account (Eq. (4)) for $d_{p}=10 \mu \mathrm{m}$ and $d_{p}=100 \mu \mathrm{m}$ particle size. ${ }^{10}$ All the experimental points are above the limit set by Eq. (4) for their particle size. For experiments in which the collected mass $m_{d}$ was available (filled symbols), the particle charge $q_{d}$ was calculated using the dispersed mass $m_{d}$ rather than the collected mass $m$. experimental point are given in the figure caption. From the results of Fig. 3, changing the storage humidity from $60 \%$ to $30 \%$ does not greatly influence the charge acquired by the particles in dispersion $q_{d}$. The only available data with dry $\mathrm{N}_{2}$ and $10 \% \mathrm{RH}$ were obtained with $5-50 \mu \mathrm{m}$ glass beads. Finally, the fact that the experimental values of the particle charge $q_{d}$ lay close to the limit imposed by corona discharge, irrespective of the nature of the particle material, indicates that the details of the contact charging between the particles and the steel tribocharger are erased by the corona discharge of particles to the surrounding gas, as proposed by Ref. 10 .

An isolated particle, however, represents an idealized case since dispersed particles in the gas stream are not immersed in an infinite volume of gas but surrounded by other particles contained in the same volume and similarly charged. Inside a conductive tube, the maximum charge over a particle is lower than for the case of an isolated particle due to the influence of the charges in neighbouring particles and the presence of image charges in the tube. According to Ref. 10, the maximum charge $q_{d, \text { max }}$ for particles of radius $r_{p}$ dispersed in the gas stream with volume fraction $\varphi$ in a conductive tube of diameter $D$ is

$$
q_{d, \max }=\frac{8.80 \times 10^{-4} r_{p}^{3}}{\sqrt{\varphi^{2} D^{2}+\left(136.8 r_{p}^{1.5}\right)^{2}}},
$$

where $q_{d, \text { max }}$ is given in coulombs and $r_{p}$ in meters. For an isolated particle, $\varphi \rightarrow 0$ and Eq. (3) yields

$$
q_{d, \max }=1.82 \times 10^{-5} r_{p}^{1.5},
$$

which in the range of particle radius $r_{p}$ between 10 and $100 \mu \mathrm{m}$ yields values of $q_{p, \text { max }}$ similar to those given by Eq. (2).

In Fig. 4, we have plotted the values of the electric $q_{d}$ acquired from the steel tube tribocharger by particles in suspension as a function of the volume fraction $\varphi$ of the particles, calculated from the gas flow rate in the outside port of the venturi $G$ (in $\mathrm{sccm} / \mathrm{s}$ ), the average mass flow rate $m / t_{\text {exp }}$ (in $\mathrm{g} / \mathrm{s}$ ) at which the powder is collected in the Faraday pail ( $t_{\text {exp }}$ is the duration of the experiment) and the particle density $\rho_{p}\left(\mathrm{~g} / \mathrm{cm}^{3}\right)$. When data are available, the mass flow rate into the venturi has been substituted in $m / t_{\exp }$

$$
\varphi=\frac{1}{G \rho_{p}} \frac{m}{t_{\exp }} .
$$

In Fig. 4, the values of the particle charge $q_{d}$ are under the limit given by Eq. (3) because the volume fraction $\varphi$ of the particles in the dispersing gas stream is not large enough to allow the electric charge on each particles influence significantly the electric charge on neighboring particles. For the volume fractions at which we have dispersed the powders, Eq. (3) predicts that there should be an effect of neighboring particles on the maximum electric charge for particles of diameter less than $10 \mu \mathrm{m}$. All the powders charged with the steel tube tribocharger have particle sizes larger than $10 \mu \mathrm{m}$, and therefore for the conditions tested, their particle charges are not affected by the presence of neighboring particles, but these may be important issues when testing finer materials. 
For one of the materials tested, $(5-50 \mu \mathrm{m})$ glass beads, we have recorded the particles trajectories as they exit the steel tube tribocharger using a high-speed camera (Phantom Miro310). For recording these images, the steel tube tribocharger was connected to a fiberglass extension with glass windows that allowed to see in the direction perpendicular to the particle laden gas flow. A high speed camera was fitted with magnification optics yielding an image scale of approximately $7 \mu \mathrm{m} /$ pixel. Back illumination was provided by a high intensity LED during the recording time. In the fiberglass extension, two parallel electrodes connected to a function generator and a high voltage amplifier (model 20/20 A, Trek, Inc.) created an oscillating electric field perpendicular to both the gas flow and the viewing direction. The electrodes were located in the outside of the fiberglass extension, so the particles could not have direct contact with the electrodes. The camera recording is synchronized with the electric field so the phase of the field is known in the recordings. For recording the images, a short gas pulse $(0.3-1 \mathrm{~s})$ is used to drive the particles through the tribocharger. Short pulses are necessary to allow the particles to slow at the exit of the tribocharger down to a speed that allows trajectories to be tracked in the images at the used frame rate (4000 frames per second).

The electric charge of each particle is obtained from the analysis of particle trajectories when the particles pass through a region occupied by the alternating electric field. Charged particles oscillate in the field, and the value of their charge and its sign can be obtained from the oscillation amplitude and phase lag with the field using the procedure described in Ref. 15. The results for a total of 330 trajectories are shown in Fig. 5. In this figure, we present the particle surface charge density $\sigma$ as a function of particle radius $R$ as well as the particle size distribution for the trajectories recorded and the polarity of the particle charges. Each

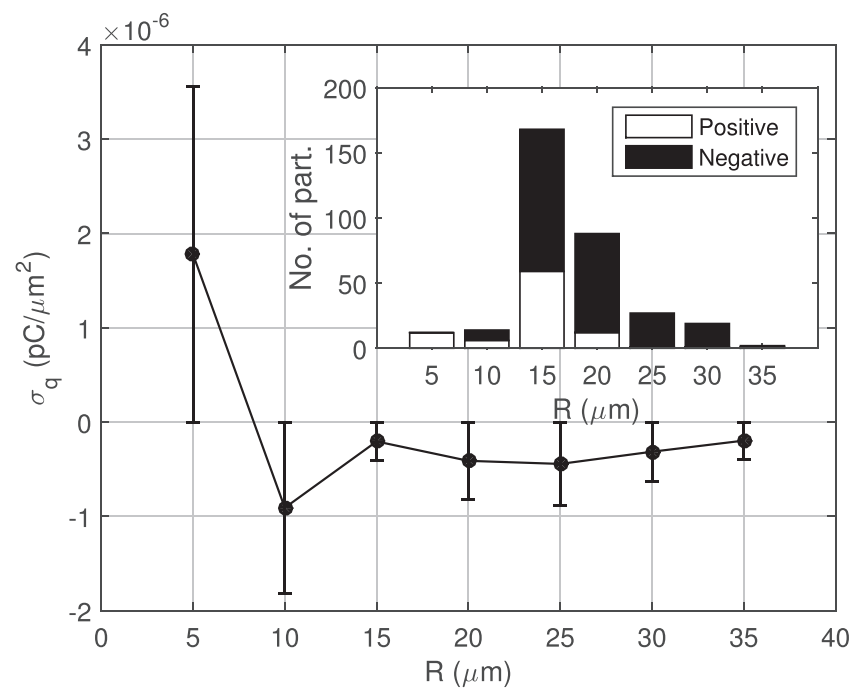

FIG. 5. Dependence of the surface charge density $\sigma_{q}$ on the particle radius for $5-50 \mu \mathrm{m}$ glass beads charged in the steel tube tribocharger. The electric charge and the radius of individual particles have been measured from the analysis of the particle trajectories recorded by a high-speed camera as the particles leave the tribocharger. The inset displays the particle size distribution of the imaged particles and the sign of the charge carried by the particles. particle radius was measured from the particles that are in the recorded images.

Most particles charged negatively, i.e., with the same size of the dispersed powder after leaving the tribocharger, but interestingly, some of the smaller particles are positively charged. The plot of the surface charge density of the particles $\sigma$ as a function of the particle radius shows that $\sigma$ is more or less constant for the larger particles of the sample, as it would be if these particles would have attained their maximum charge, but deviates for the smaller particles. The deviation in the value of $\sigma$ for the smaller particles may be explained if these particles, being able to follow the gas flow lines better than the larger particles, experienced fewer collisions with the tribocharger walls and still maintained, at least partially, the electric charge distribution they had before the sample was dispersed, which is expected to expand both signs as it is the result of charge transfer between particles. ${ }^{16}$

\section{B. Charge per particle in settled powder}

We have seen that the charge transfer process in the steel tube tribocharger produces the same charge $q_{d}$ in all the particles if we assume the sample is monodisperse. If the particles did not discharge during collection of the sample into a settled powder, the charge $Q_{s}$ in a settled sample would equal the total charge $Q_{d}$ transferred to its particles in the steel tube tribocharger. However, this is not so. In fact, if we plot $Q_{s}$ as a function of the collected mass $m$ as we have done in Fig. 6 for the same experiment, as shown in Fig. 2, in all cases, $Q_{s}<Q_{d}$. Moreover, the plot of $Q_{s}$ versus collected mass $m$ always shows a sublinear dependence of $Q_{s}$ on $m$. Since the charge to mass ratio $q m r$ of the collected sample is the derivative of the curve of $Q_{s}$ vs. $m$, this fact means that the last parts of the sample to be collected retain less charge than the first parts. In the following parts of the manuscript, whenever we refer to the specific charge of the collected sample $q m r$, we

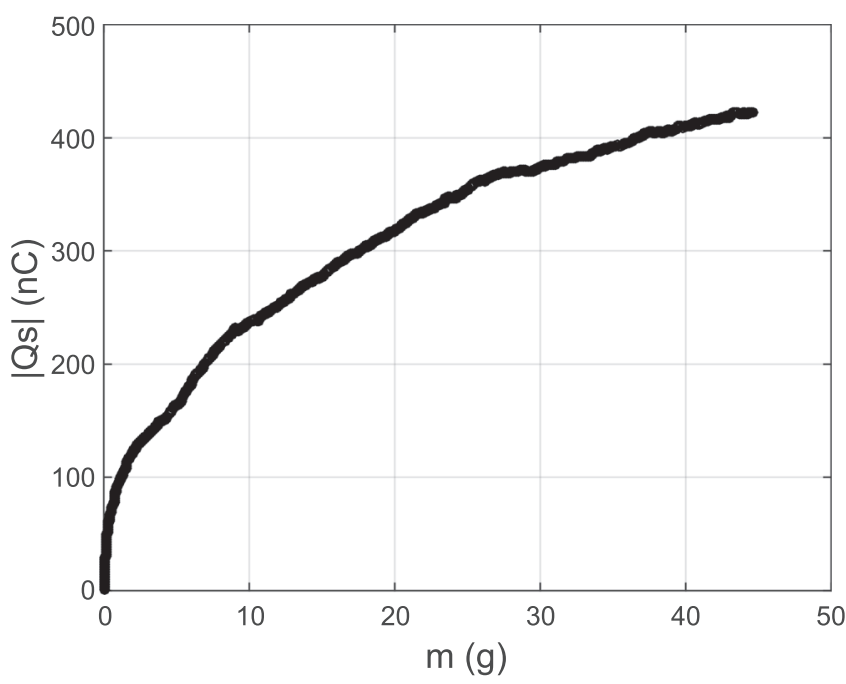

FIG. 6. Electric charge $Q_{s}$ in the collected sample as a function of the collected mass for the same experiment as in Fig. 4. The sublinear dependence of $Q_{s}$ with the mass $m$ indicates that the collected powder is not charged uniformly. Note that the collected charge $Q_{s}$ is about $1 / 100$ of the charge $Q_{d}$ acquired by the particles in the tribocharger. 
will refer to its average value obtained by dividing the final value of $Q_{s}$ against the total collected mass $m$.

Fig. 7 illustrates the specific charge $q m r$ of the collected powder against the collected mass $m$ for all the available experiments, that is, using the steel tube and the nylon cyclone tribochargers. For a given material, the specific charge $q m r$ tends to decrease when more mass is collected in agreement with the sublinear dependence observed for the charge $Q_{s}$ in the collected mass $m$ shown in Fig. 6. Although the data points presented in Fig. 7 have a large scatter, there is a visible tendency of decreasing specific charge $q m r$ with sample mass $m$. Moreover, data obtained with different tribochargers with the same material follow the same trend, indicating that the mechanisms that limit the electric charge in the dispersed particles and the collected sample are the same for both types of tribochargers, although the total charge $Q_{d}$ transferred to the particles when suspended in the gas stream cannot be measured for the nylon cyclone.

The average electric charge per particle in the collected sample can be calculated from $q_{s}=Q_{s} m_{p} / m$. For the experiments with the steel tube tribocharger, $q_{s}$ can be compared with the electric charge per particle when the sample was dispersed in the gas stream $q_{d}$ to evaluate the amount of charge lost during settling. Such a comparison is done in Fig. 8 and shows that in most cases $q_{s}$ is between 1/100 and $1 / 10$ of the charge $q_{d}$ acquired from the tribocharger.

\section{DISCUSSION}

To explain the discharge of the particles when they settle, we assume that there is a corona discharge from the surface of the settled sample to the surrounding air and that the remaining charge is determined by the condition that the electric field at the surface of the sample must equal the electric field for corona discharge in air, which we take for simplicity as $E_{c}=3 \times 10^{6} \mathrm{~V} / \mathrm{m}$, although it should have some

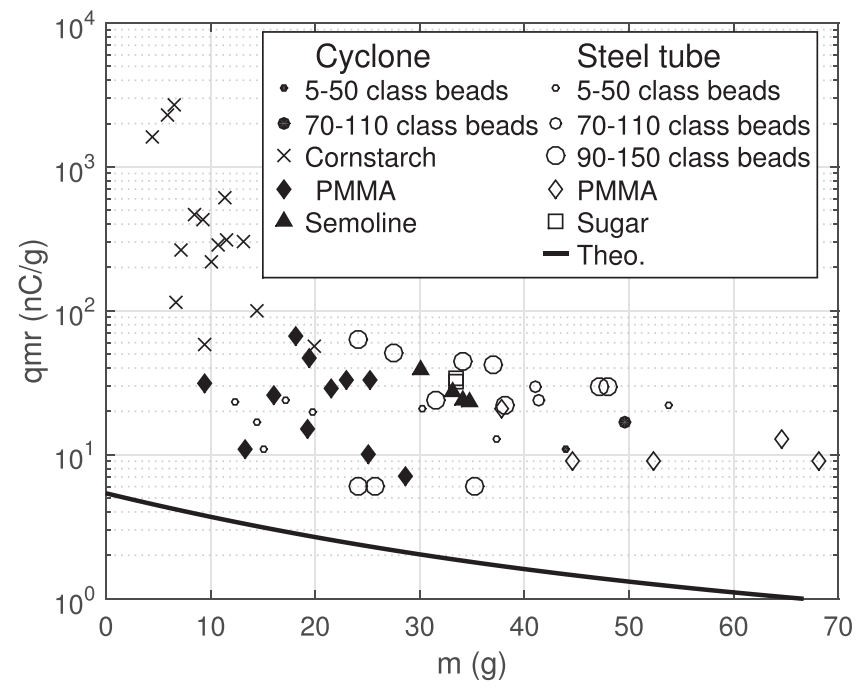

FIG. 7. Specific charge $q m r$ (charge to mass ratio) of the collected sample as a function of the collected mass $m$. Data for all the experimental runs are included. The void symbols represent data from experiments using the steel tube tribocharger. Data with filled symbols represent data from the experiments using the nylon cyclone tribocharger. The solid line represents the result of the model presented in Sec. V for PMMA.

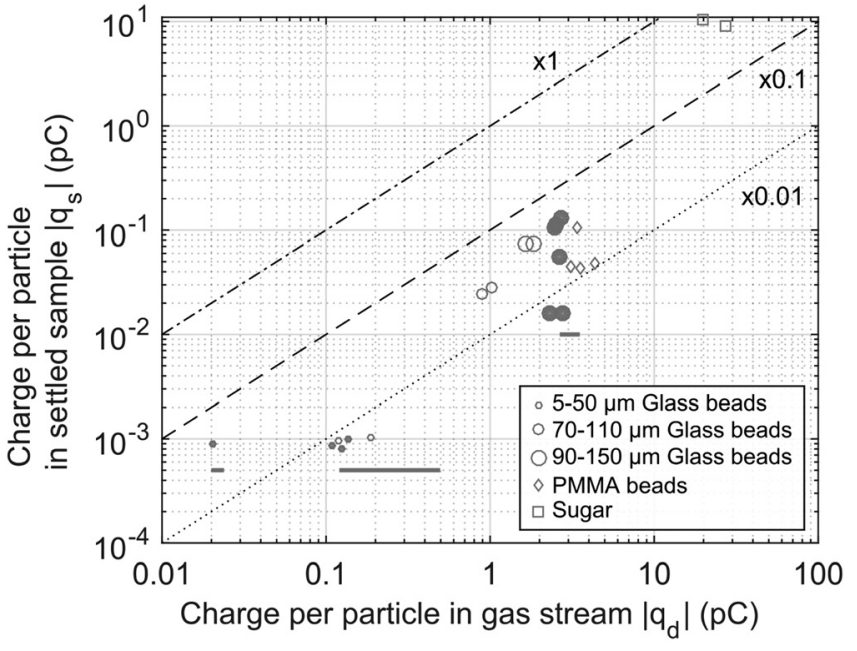

FIG. 8. Electric charge per particle $q_{s}$ in the collected sample versus the electric charge per particle $q_{d}$ in the dispersed sample for the samples tested using a steel tube tribocharger. For experiments in which the collected mass $m_{d}$ was available (filled symbols), the particle charge $q_{d}$ was calculated using the dispersed mass $m_{d}$ rather than the collected mass $m$. Lines are the cases when $q_{s}$ is equal to $1,0.1$, and 0.01 times $q_{d}$.

dependence on the sample size. ${ }^{1}$ Since the calculation of the electric field created by a cylindrical heap of powder is not straightforward, for the sake of gaining insight on the problem first, we will discuss a simplified problem in which the sample is assumed to be an infinite layer in the $X Y$ plane that grows in the positive $Z$ direction by uniform addition of particles to its surface. Both the real geometry of our setup and the geometry of the simplified problem are depicted in Fig. 9. The layer rests on a conductive plate representing the metallic filter of the collecting cell. The mass flow rate of new particles per unit area is $F_{m}$, so if the mass density of the powder layer is $\rho_{m}$, the height $H$ of the powder layer grows as

$$
H=\frac{F_{m}}{\rho_{m}} t,
$$

until at $t=t_{f}$ the sample collection stops and the powder layer attains its final depth $H_{f}$. Modeling the details of the corona discharge from the powder is complicated and we will

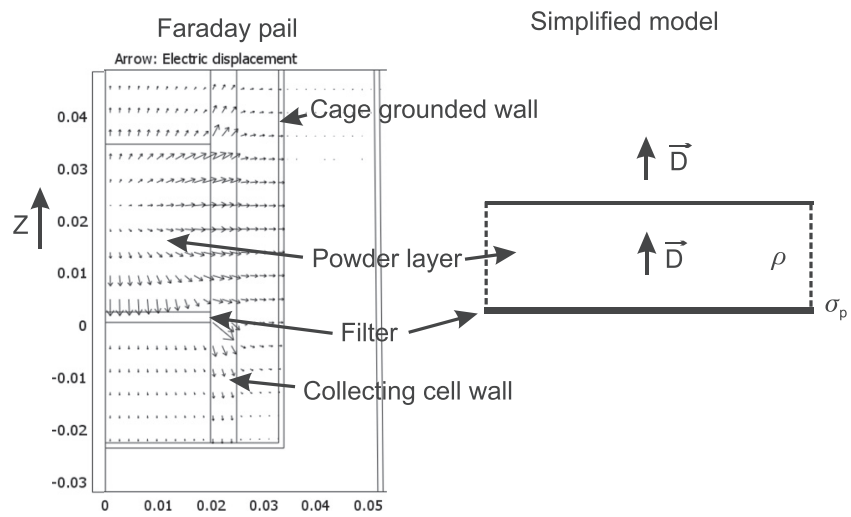

FIG. 9. COMSOL model of a charged powder layer inside the collecting cell and Faraday cage used in our experiments. Coordinates in the diagram are given in meters. To the right, the simplified model discussed in Section $\mathrm{V}$. Arrows in the COMSOL model indicate the magnitude and the direction of the electric field displacement if there were no discharge in the powder. 
re-sort to the simplifying assumption that the powder layer has an electrical conductivity $\sigma$. This electrical conductivity represents the ability of the electric charge inside the powder layer to move once the corona discharge is initiated. In rigorous terms, $\sigma$ should only have a nonzero value once the corona discharge is triggered, but in this analysis, we will assume $\sigma$ as a constant value independent of time. The temporal evolution of the charge per unit volume $\rho(z, t)$, the electric displacement $D(\vec{z}, t)$ inside the layer as well as the surface charge density on the conductive plate $\sigma_{p}(t)$ are given by the solution of the set of equations

$$
\begin{gathered}
\nabla \cdot \vec{D}=\rho+\sigma_{p} \delta(z), \\
\frac{\partial \rho}{\partial t}+\nabla \cdot \vec{j}=\rho_{o} \delta\left(t-z \frac{\rho_{m}}{F_{m}}\right),
\end{gathered}
$$

$$
\frac{d \sigma_{p}}{d t}=-j_{z}(0, t)=-\frac{\sigma}{\epsilon} D_{z}(0, t),
$$

where $\delta\left(t-z \rho_{m} / F_{m}\right)$ is the Dirac's delta function and $\rho_{o}$ represents the charge per unit volume on a newly deposited layer of powder. Due to the geometry of the problem, the spatial derivatives are given by $\nabla=\vec{u}_{z} \partial /(\partial z)$, the electric displacement by $\vec{D}=D_{z} \vec{u}_{z}$, and the current density by $\vec{j}=\sigma D_{z} / \epsilon \vec{u}_{z}$. As the powder losses its charge, the charge per unit volume $\rho(z, t)$ decreases from the value $\rho_{o}$ : a part of the charge is lost to the surrounding air by corona discharge and a part migrates to the metallic filter. As initial conditions, we take that the initial height of the powder layer to be zero and that the plate representing the filter is discharged.

The solution of Eq. (7) is

$$
\begin{aligned}
\rho(z, t) & =\rho_{o} \exp \left[-\frac{\sigma}{\epsilon}\left(t-\frac{\rho_{m}}{F_{m}} z\right)\right] \Theta\left(t-\frac{\rho_{m}}{F_{m}} z\right) \quad t \leq t_{o} \\
D_{z}(z, t) & =\rho_{o} \frac{\epsilon}{\sigma} \frac{F_{m}}{\rho_{m}}\left\{\exp \left[-\frac{\sigma}{\epsilon}\left(t-\frac{\rho_{m}}{F_{m}} z\right)\right]-\exp \left(-\frac{\sigma}{2 \epsilon} t\right)\right\} \\
\sigma_{p}(t) & =\rho_{o} \frac{\epsilon}{\sigma} \frac{F_{m}}{\rho_{m}}\left[1+\exp \left(-\frac{\sigma}{\epsilon} t\right)-2 \exp \left(-\frac{\sigma}{2 \epsilon} t\right)\right] t>t_{0} \\
D_{z}(z, t) & =\rho_{o} \frac{\epsilon}{\sigma} \frac{F_{m}}{\rho_{m}}\left\{\exp \left[-\frac{\sigma}{\epsilon}\left(t-\frac{\rho_{m}}{F_{m}} z\right)\right]-\exp \left[-\frac{\sigma}{\epsilon}\left(t-t_{o}\right)\right]+\exp \left[-\frac{\sigma}{2 \epsilon}\left(t-t_{o}\right)\right]-\exp \left(-\frac{\sigma}{2 \epsilon} t\right)\right\} \\
\sigma_{p}(t) & =\rho_{o} \frac{\epsilon}{\sigma} \frac{F_{m}}{\rho_{m}}\left\{2 \exp \left[-\frac{\sigma}{2 \epsilon}\left(t-t_{o}\right)\right]\left[1-\exp \left(-\frac{\sigma}{2 \epsilon} t_{o}\right)\right]+\exp \left[-\frac{\sigma}{\epsilon}\left(t-t_{o}\right)\right]\left[\exp \left(-\frac{\sigma}{\epsilon} t_{o}\right)-1\right]\right\} .
\end{aligned}
$$

The temporal evolution of the charge density and the electric field displacement are shown in Fig. 10. As the powder layer grows, the deeper layers discharge while the upper layers remain charged with the result that only the portions of the
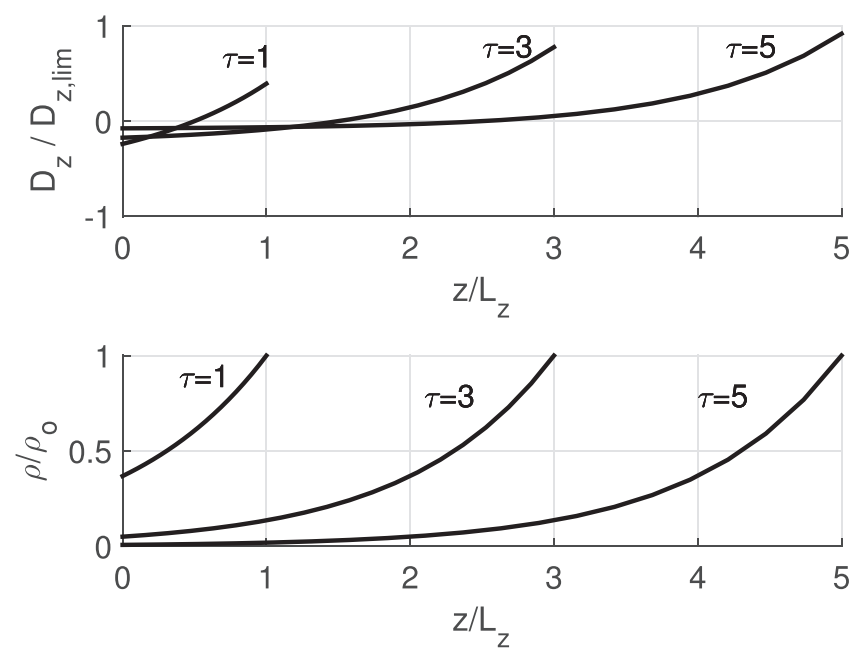

FIG. 10. Temporal evolution of the electric field displacement $D_{z}$ inside the powder layer and the volume charge density $\rho$ as a function of position inside the powder layer $z$ and the elapsed time $t$ since the start of the deposition of the layer. The time is measured in units of $\tau=\epsilon / \sigma$ and the $z$ coordinate in units of the height $L_{z}$ of powder deposited during a time $\tau$. powder layer close to the surface remain charged. The depth of the charge-holding layer depends both on the effective conductivity of the powder and the speed at which the powder is fed to the layer. The electric field displacement (which is proportional to the electric field) is nonzero only in the region where the powder remains charged and above the powder surface. The same pattern of charge and field distribution has found to happen during loading of silos. ${ }^{17}$ The total charge per unit area in the layer of powder is given by

$$
\begin{aligned}
& \frac{Q}{A}=\int_{o}^{H(t)} d z \rho(z, t) \Rightarrow \\
& \frac{Q}{A}=\rho_{o} \frac{\epsilon}{\sigma} \frac{F_{m}}{\rho_{m}}\left[1-\exp \left(-\frac{\sigma}{\epsilon} t\right)\right] ; \quad t \leq t_{o} \\
& \frac{Q}{A}=\rho_{o} \frac{\epsilon}{\sigma} \frac{F_{m}}{\rho_{m}} \exp \left[-\frac{\sigma}{\epsilon}\left(t-t_{o}\right)\right]\left[1-\exp \left(-\frac{\sigma}{\epsilon} t_{o}\right)\right] ; \quad t \leq t_{o} .
\end{aligned}
$$

While the powder layer is growing $t \gg \epsilon / \sigma$, the electric displacement on the surface of the powder layer tends to the value

$$
D_{z}(H, t) \rightarrow \rho_{o} \frac{\epsilon}{\sigma} \frac{F_{m}}{\rho_{m}}=D_{z, \text { lim }} .
$$


The electric field outside the powder layer is given by $E_{z}=D_{z}(H, t) / \epsilon_{o}$. If we identify the value of the electric field obtained from Eq. (12) with the electric field $E_{c}=30 \mathrm{kV} / \mathrm{cm}$ for corona discharge on air, we get

$$
E_{c}=\rho_{0} \frac{\epsilon}{\epsilon_{o}} \frac{1}{\sigma} \frac{F_{m}}{\rho_{m}},
$$

where the conductivity of the layer $\sigma$ and the initial charge density on a newly deposited powder layer $\rho_{o}$ must be evaluated from experimental data, provided we use can write another equation in which both quantities are involved. We get a second equation from the temporal evolution of the total charge in the Faraday pail $Q / A+\sigma_{p}$. While the powder layer is growing, the total charge per unit area is given by

$$
\frac{Q}{A}+\sigma_{p}=2 \rho_{o} \frac{\epsilon}{\sigma} \frac{F_{m}}{\rho_{m}}\left[1-\exp \left(-\frac{\sigma}{2 \epsilon} t\right)\right],
$$

and once no more new powder is deposited on the powder layer, the total charge decays as

$$
\frac{Q}{A}+\sigma_{p}=\left(\frac{Q}{A}+\sigma_{p}\right)_{t_{o}} \exp \left[-\frac{\sigma}{2 \epsilon}\left(t-t_{o}\right)\right],
$$

where the value of $\left(Q / A+\sigma_{p}\right)_{t_{o}}$ is obtained by substituting $t_{o}$ in Eq. (14). The temporal evolution of the total charge inside the Faraday pail is represented in Fig. 11. During the initial stages of growth of the powder layer, the total charge grows sublinearly with time, resembling the dependence of the collected charge on collected mass depicted in Fig. 6, since in our experiments the mass is collected at a constant rate and thus it is proportional to time.

Once the powder layer stops growing, the total charge in the Faraday pail decays as $\exp (-\sigma t /(2 \epsilon))$ (see Eq. (15)). This means we can estimate the value of $\tau=2 \epsilon / \sigma$, if we measure the rate of dissipation of the charge in our samples. We have done this for PMMA beads (see Fig. 12) with the result that $\tau=61 \pm 17 \mathrm{~s}$. In order to get an estimate for the effective layer conductivity $\sigma$, we need a value for the electric permittivity of the powder $\epsilon$. The dielectric constant

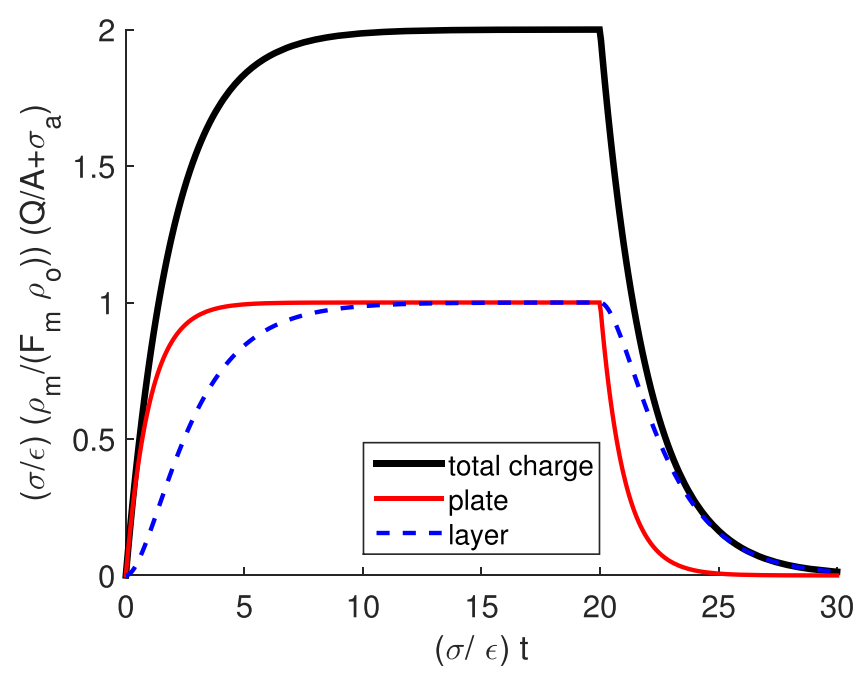

FIG. 11. Temporal evolution of the total charge in the Faraday pail.

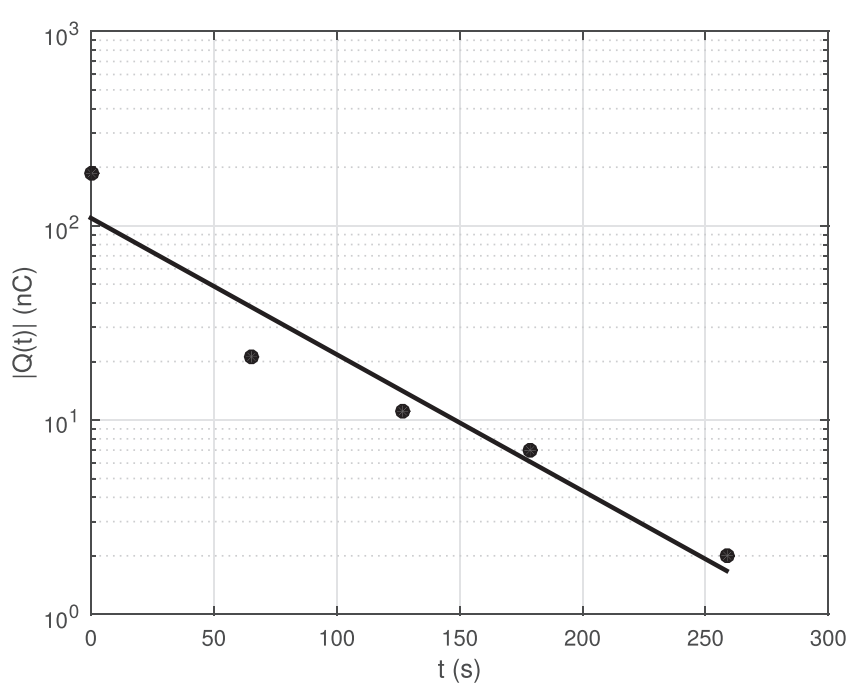

FIG. 12. Temporal evolution of the total charge in a sample of PMMA after collection. The straight line is a fit to an exponential decay.

$k=\epsilon / \epsilon_{o}$ of PMMA ranges between 2.8 and 4. According to the Bruggeman mixing formula ${ }^{18}$ for a solid fraction $\phi=0.6$ for the powder, the values that correspond to the limits of the range are $k=1.94$ and $\mathrm{k}=2.50$ so we take a value of $k=2.22 \pm 0.28$ for the dielectric constant of the layer; so, $\sigma=2 k \epsilon_{o} / \tau=(5.5 \pm 1.6) \times 10^{-9} \mathrm{~S} / \mathrm{m}$. The density of solid PMMA is $\rho_{P}=1.15-1.19 \mathrm{~g} / \mathrm{cm}^{3}$. Assuming a solid fraction of $\phi=0.6$, this yields $\rho_{m} \simeq 0.70 \mathrm{~g} / \mathrm{cm}^{3}$. The rate of discharge of the powder in the experiments with PMMA is about $0.20 \mathrm{~g} / \mathrm{s}$. For a cell of $4 \mathrm{~cm}$ in diameter yields $F_{m}$; so, we can estimate the charge per unit volume of a newly deposited layer of powder as

$$
\rho_{o}=E_{c} \frac{\sigma}{k} \frac{\rho_{m}}{F_{m}}
$$

which yields $\rho_{o}=3.3 \mathrm{nC} / \mathrm{cm}^{3}$ which for $\rho_{m}=0.70 \mathrm{~g} / \mathrm{cm}^{3}$ is equivalent to $q m r=4.7 \mathrm{nC} / \mathrm{g}$, which is of the same order of magnitude than the results obtained for the specific charge in the collected sample $(q m r=10-20 \mathrm{nC} / \mathrm{g})$.

If the values obtained for $\rho_{o}, \sigma$, and $\epsilon$ are substituted in the equation for the total charge per unit area in the Faraday pail as a function of time (Eq. (14)) and we use that the section of the collecting cell is $12.56 \mathrm{~cm}^{2}$, we can obtain a prediction of the specific charge $q m r$ as a function of the collected mass $m$. The resulting curve is plotted in Fig. 7, where it can be compared with the experimental data for PMMA beads. The model seems to give the correct trend in the data of specific charge against collected mass, although the values of the specific charge are about an order of magnitude smaller than the experimental values. The difference between the predictions of the model and the experimental results arises from two reasons. The first one is that we have assumed that the effective electrical conductivity of the powder $\sigma$ is nonzero since the start of powder collection, whereas in the real powder, it might be zero until the electric field on the surface of the powder has risen to breakdown field in air, and thus, in the initial stages of powder collection, the charge does not dissipate. The second reason is that the planar geometry of the 
model is expected to yield higher electric fields than the real geometry, and therefore, the breakdown field in air on the surface of the layer is reached with less amount of charge than in the real geometry.

\section{CONCLUSIONS}

Our experimental data support the fact that the maximum electric charge a particle can hold in dilute suspension is limited by the breakdown electric field of air, rather than by the microscopical mechanisms driving the charge transfer between the particles and the solid surfaces from where the charge is transferred. When charged particles settle to form a packed powder, deposition of the particles is accompanied by a corona discharge of the settled powder. This discharge has the consequence that the specific charge (charge per unit mass) of the settled powder decreases with collected mass. However, according to a simple onedimensional model of the powder deposition and discharge that successfully predicts the trend of specific charge to collected mass, the charge in the powder is concentrated in the layers close to the powder surface on which the electric field is close to the breakdown field for corona discharge on air. From the electric field distribution of the model, the electric energy stored in a settled powder could be calculated, yielding an estimate of the maximum energy of a possible electrical discharge which can be useful to determine the risks posed by electrostatic charge accumulation during powder settling for a given mass flow rate of powder and electric charge on the dispersed particles. However, to be fully predictive, the model would require an independent measurement of the powder effective conductivity such as those proposed in Ref. 19.

\section{ACKNOWLEDGMENTS}

This project has been funded by the Spanish Ministerio de Economía y Competitividad (Project No. FIS2014-54539P) and by the International Fine Particle Research Institute (IFPRI). Particle size distribution of the materials to determine their mean particle sizes was measured at the Centro de Investigación Tecnología e Innovación de la Universidad de Sevilla (CITIUS). We would like to dedicate this manuscript to the memory of Antonio Castellanos, who passed away while this manuscript was in preparation.

${ }^{1}$ W. Harper, Contact and Frictional Electrification (Oxford University Press, 1967).

${ }^{2}$ J. Lowell and A. Rose-Innes, Adv. Phys. 29, 947 (1980).

${ }^{3}$ L. B. Schein, J. Electrost. 46, 29 (1999).

${ }^{4}$ S. Matsusaka and H. Masuda, Adv. Powder Technol. 14, 143 (2003).

${ }^{5}$ A. Díaz, J. Adhes. 67, 111 (1998).

${ }^{6}$ V. Albrecht, A. Janke, E. Németh, S. Spange, G. Schubert, and F. Simon, J. Electrost. 67, 7 (2009).

${ }^{7}$ M. Apodaca, P. Wesson, K. Bishop, M. Ratner, and B. Grzybowski, Angew. Chem. 122, 958 (2010).

${ }^{8}$ T. Matsuyama and H. Yamamoto, Chem. Eng. Sci. 61, 2230 (2006).

${ }^{9}$ J. Lowell and W. Truscott, J. Phys. D: Appl. Phys. 19, 1273 (1986).

${ }^{10}$ T. Matsuyama and H. Yamamoto, Adv. Powder Technol. 21, 350 (2010).

${ }^{11}$ M. Glor and K. Schwenzfeuer, J. Electrost. 40-41, 511 (1997).

${ }^{12}$ B. Maurer, M. Glor, G. Luttgens, and L. Post, J. Electrost. 23, 25 (1989).

${ }^{13}$ X. Meng, H. Zhang, and J. Zhu, J. Phys. D: Appl. Phys. 41, 195207 (2008).

${ }^{14}$ P. M. Ireland and G. J. Jameson, J. Electrost. 71, 449 (2013).

${ }^{15}$ M. Espin, J. Valverde, M. Quintanilla, and A. Castellanos, Phys. Rev. E 79, 011304 (2009).

${ }^{16}$ K. Forward, D. Lacks, and R. Sankaran, Phys. Rev. Lett. 102, 028001 (2009).

${ }^{17}$ W. Azizi, J. Phys.: Conf. Ser. 646, 012046 (2015).

${ }^{18}$ K. Kärkkäinen, A. Sihvola, and K. Nikoskinen, IEEE Trans. Geosci. Remote Sens. 39, 1013 (2001).

${ }^{19}$ Y. Aleksin, A. Vora, and U. Riebel, Powder Technol. 294, 353 (2016). 